tor or receptors. A direct binding partner of $\mathrm{PD}-1 \mathrm{H}$ has yet to be shown, and a putative receptor remains to be identified.

Third, notwithstanding the expression of PD-1H on T cells, most data to date can be accounted for by evoking PD-1H strictly as an inhibitory ligand, as previously suggested by Ceeraz et al. (16). This interpretation, while advantageous for its simplicity, fails to account for the existence of the large and very unique intracellular domain of the molecule. Wang et al. noted that VISTA is highly conserved in organisms ranging from fungi to humans (5); however, while the extracellular IgV domain of VISTA shows homology with $\mathrm{B} 7 \mathrm{H} 1$, no relative of the large intracellular domain has been found, suggesting that this intracellular domain may have evolved independently of other cosignaling molecules. The unusual evolutionary conservation of the intracellular domain of $\mathrm{PD}-1 \mathrm{H}$ hints at a functional importance for this part of the molecule, but its uniqueness shields any clue as to what that function may be. With these outstanding issues and the strong effect of targeting PD- $1 \mathrm{H}$ in transplantation, autoimmune diseases, and cancer, it is safe to assume that we have not heard the last from this protein.

\section{Acknowledgments}

I thank Pan Zheng and Dashuang Shi for helpful discussion. This work is supported by grants from the NIH (CA58033 and AI64350).

Address correspondence to: Yang Liu, Centers for Cancer and Immunology Research, Children's Research Institute, Children's National Medical Center, 111 Michigan Avenue NW, Washington, DC 20010, USA. Phone: 202.476.5849; Fax: 202.476.6498; E-mail: yaliu@cnmc.org.

1. Genovese MC, et al. Abatacept for rheumatoid arthritis refractory to tumor necrosis factor $\alpha$ inhibition. N Engl J Med. 2005;353(11):1114-1123.

2. Hwu P. Treating cancer by targeting the immune system. N Engl J Med. 2010;363(8):779-781.

3. Kremer JM, et al. Treatment of rheumatoid arthritis by selective inhibition of T-cell activation with fusion protein CTLA4Ig. N Engl J Med. 2003 ; 349(20):1907-1915

4. Ribas A. Tumor immunotherapy directed at PD-1. NEngl J Med. 2012;366(26):2517-2519.

5 . Wang L, et al. VISTA, a novel mouse Ig superfamily ligand that negatively regulates $\mathrm{T}$ cell responses. J Exp Med. 2011;208(3):577-592.

6. Flies DB, Wang S, Xu H, Chen L. Cutting edge: A monoclonal antibody specific for the programmed death-1 homolog prevents graft-versus-host disease in mouse models. J Immunol. 2011;187(4):1537-1541.

7. Bachmann MF, Gallimore A, Jones E, Ecabert B, Acha-Orbea H, Kopf M. Normal pathogen-specific immune responses mounted by CTLA-4-deficient T cells: a paradigm reconsidered. Eur J Immunol. 2001; 31(2):450-458.

8. Bachmann MF, Kohler G, Ecabert B, Mak TW, Kopf M. Cutting edge: lymphoproliferative disease in the absence of CTLA- 4 is not T cell autonomous. JImmunol. 1999;163(3):1128-1131.

9. Liu Y. Is CTLA-4 a negative regulator for T-cell activation? Immunol Today. 1997;18(12):569-572.

10. Nguyen TV, Ke Y, Zhang EE, Feng GS. Conditional deletion of Shp2 tyrosine phosphatase in thymocytes suppresses both pre-TCR and TCR signals. Jimmunol. 2006;177(9):5990-5996.

11. Flies DB, et al. Coinhibitory receptor $\mathrm{PD}-1 \mathrm{H}$ preferentially suppresses $\mathrm{CD}^{+} \mathrm{T}$ cell-mediated immunity. J Clin Invest. 2014;124(5):1966-1975.

12. Guerder S, Matzinger P. A fail-safe mechanism for maintaining self-tolerance. J Exp Med. 1992; 176(2):553-564

13. Ridge JP, Di Rosa F, Matzinger P. A conditioned dendritic cell can be a temporal bridge between a $\mathrm{CD}^{+} \mathrm{T}$ - helper and a T-killer cell [see comments]. Nature. 1998;393(6684):474-478.

14 . Wing K, et al. CTLA-4 control over Foxp $3^{+}$regulatory T cell function. Science. 2008;322(5899):271-275.

15. Janeway CA. Autoimmune disease: immunotherapy by peptides? Nature. 1989;341(6242):482-483.

16. Ceeraz S, Nowak EC, Noelle RJ. B7 family checkpoint regulators in immune regulation and disease. Trends Immunol. 2013;34(11):556-563.

17. Liu Y, Janeway CA, Janeway CA Jr. Interferon $\gamma$ plays a critical role in induced cell death of effector T cell: a possible third mechanism of self-tolerance. J Exp Med. 1990;172(6):1735-1739.

18. Schwartz RH. Costimulation of T lymphocytes: the role of CD28, CTLA-4, and B7/BB1 in interleukin-2 production and immunotherapy. Cell. 1992; 71(7):1065-1068

\title{
Cryptococcosis: a model for the understanding of infectious diseases
}

\author{
John R. Perfect \\ Department of Medicine, Division of Infectious Diseases, Duke University Medical Center, Durham, North Carolina, USA.
}

\begin{abstract}
The increase in immunosuppressed patient populations has correlated with a rise in clinical fungal infections, including cryptococcosis. Patient outcome following Cryptococcus infection is linked to initial fungal burden in cerebrospinal fluid (CSF) and fungal clearance following treatment; however, the role of the pathogen in disease prognosis is poorly defined. In this issue of the JCI, Sabiiti and colleagues have directly correlated phenotypic traits of Cryptococcus neoformans with clinical outcome of infected patients. A better understanding of both the host and pathogen contributions to disease etiology will provide more options for targeted treatment strategies.
\end{abstract}

\section{Clinical impact}

Cryptococcosis, which results from infection by Cryptococcus neoformans and Cryptococcus gattii, exploded onto the clinical

Conflict of interest: John R. Perfect has served as a consultant for or been on the advisory committee of Astellas, Pfizer, F2G, Viamet, and Scynexis.

Citation for this article: $J$ Clin Invest. 2014; 124(5):1893-1895. doi:10.1172/JCI75241. scene in three major outbreaks. In 1978, Kauffman and Blumer marked the first outbreak as the "awakening giant of mycology" (1). The frequency of cryptococcosis rose in concert with an enlarging immunosuppressed population as the result of advanced medical therapies for serious underlying diseases. The rapid increase in cryptococcosis was the "canary in the coal mine" sentinel finding for the fragility of immunosuppressed patients and our lack of precision in manipulating the immune system. The second major outbreak occurred in the mid-1980s as the immunosuppressed HIV pandemic gripped the world, with the number of patients affected and the effects of the virus on immunity reflected in the appearance of an incredible number of cryptococcal cases, estimated at 1 million per year worldwide (2). The third major outbreak of cryptococcosis occurred around 2000 in the Pacific Northwest as the result of C. gattii infections. This outbreak has challenged our understanding of new recombinant (hypervirulent) strains (3) and of the potential influences of climate on changing fungal ecology. Cryptococcus has become a "new-age" pathogen that reaches every level of clini- 
cal practice in both resource-limited and resource-available health care systems. It is no longer a medical oddity, since it is prominently centered in the differential diagnosis for immunocompromised patients throughout the world.

\section{The host side}

Humans function according to the "Goldilocks" paradigm for the proper immune state: either too little or too much of an immune response to an invading microbe can allow disease; therefore, the host must get the immune response "just right" to prevent pathogens from producing disease (4). Clinically, it is clear that several host-associated risk factors, including immunosuppression from corticosteroid treatment, immunobiology modifier administration, HIV infection, host genetic background (5), and even the development of autoantibodies (6), allow the development of cryptococcosis. Furthermore, due to the unique ability of cryptococcus to invade the CNS, any dysfunction in the host immune response to infection can be fatal. In fact, an overly aggressive immune reaction, such as immune reconstitution inflammatory syndrome (IRIS), has become a prominent clinical feature in cryptococcosis (7). In patients ranging from those with HIV on antiretroviral therapy, to transplant recipients undergoing pharmacological immunosuppression, to apparently normal hosts, IRIS must be diagnosed and managed by clinicians caring for patients with cryptococcal meningitis. Unfortunately, the current guidelines for IRIS identification and treatment are less than robust; therefore, it is the "art of the clinician at the beside" that presently makes a difference in IRIS outcome.

\section{The pathogen}

The clinical understanding of cryptococcosis has been primarily focused on the host side of this disease process, with how the patient responds to the pathogen being the critical determinant for treatment. In this issue of the JCI, Sabiiti et al. (8) have provided evidence that the phenotypic traits of the infecting strain of C. neoformans influences clinical outcome. This study by Sabiiti and colleagues provides a reality check; infections are two-way streets that are maneuvered by both host and pathogen. In elegant and precise experiments, 65 C. neoformans isolates from clinical trial patients were evaluated to determine murine mac- rophage uptake in vitro, intracellular proliferation rate, capsule induction, and laccase activity. These fungi-specific traits were then compared with the clinical data on the patient from whom the strain was isolated. Sabiiti and colleagues determined that certain features, such as yeast phagocytosis and laccase activity, correlate with disease outcome (8). Previous studies have also correlated disease manifestations with capsular production by the infecting yeast strain (9).

The association of C. neoformans phenotypes with clinical manifestations confirms that it is important to understand the principles of "how cryptococcus does it." During the past two decades, there has been a remarkable explosion in our understanding of this encapsulated pathogen at the molecular level. We have identified and linked many virulence phenotypes of the yeast, such as high-temperature growth, melanin production, and capsule formation, to their controlling genes, and we continue to delve into the molecular details of how Cryptococcus produces disease in the genome-sequencing era. Whole-genome studies are beginning to be used to identify microevolutionary events that determine strain-specific pathogenicity $(10,11)$ and to understand the plasticity of the Cryptococcus genome under stress (12). Furthermore, we can even capture how the yeast molecularly responds to the human CNS (13). Fundamentally, the molecular tools of stress adaptation to a harsh host environment are critical to cryptococcosis, and we are now beginning to broadly understand the networks this yeast uses for survival and resistance to the immune system and to therapeutic interventions. The basic Cryptococcus life cycle is not completed in the human host, but the yeast's survival characteristics are well adapted; therefore, an understanding of fungal survival principles will allow the development of therapeutic strategies that better interrupt the production of disease.

\section{The diagnosis}

Cryptococcosis vividly demonstrates two important principles for diagnostic microbiology. First, because of this fungi's ability to shed detectable polysaccharide capsules into biological fluids, the cryptococcal antigen has been one of the best serologies in infectious diseases today. Recently, a cheap, easy-to-use, very effective lateral flow assay for polysaccharide antigen detection was developed that can now be integrated into screening strategies in high-risk patients in both resourcelimited and resource-available settings (14). With this testing platform, the principle of "early therapy represents better outcome" can be realized, leaving the onus on clinicians to initiate preemptive testing. A second and more contentious area for diagnostic microbiology and cryptococcosis is the quantitation of microbial burden. In clinical infectious diseases, the practice of linking therapeutic strategies to microbial burden has infrequently been incorporated into diagnostic and therapeutic guidelines; however, two prime successful clinical examples of microbial quantitation include quantitative counts of bacteria in urinary tract infections and the effective use of quantitative viral loads in blood (such as HIV and CMV) for antiviral therapy. Cryptococcal meningitis has great potential, with further careful standardization, to provide clinicians with meaningful quantitative cryptococcal cultures (QCCs) in CSF both for prognostic and therapeutic decisions (15, 16). Although not routinely performed in clinics, QCCs have been used effectively for years in research to understand and make predictions for antifungal therapeutic outcome based on measurements of early fungicidal activity (EFA) (17). The QCC and EFA continue to be validated, and adoption of these strategies in clinical practice to better understand therapeutic responses is a worthwhile future goal that will lead to more precise management of cryptococcal meningitis.

\section{Clinical management}

The current basis for cryptococcosis management has a range of support from robust, evidence-based studies to clinical art. The 2010 Infectious Disease Society of America (IDSA) guidelines have attempted to establish consistent therapeutic principles for disease management (18). These recommendations are dynamic and will need to be modified as new insights are gained. In fact, at my own institution, we have retrospectively validated that patients treated by clinicians following IDSA recommendations likely had an improved outcome for cryptococcal meningitis (19). The immediate future is unlikely to see any improved anticryptococcal drug(s); therefore, the following principles are imperative for present therapies articulated by the IDSA 
guidelines: first, cryptococcal meningitis is treated in phases (induction, consolidation, and maintenance). Second, combination therapy with amphotericin B plus flucytosine comprises the most highly fungicidal regimen, and rapid killing of yeasts in CSF delivers a positive outcome. Third, increased intracranial pressure can create substantial morbidity and mortality; however, management of this symptom is necessary but imprecise. Fourth, development of IRIS is a factor in clinical failure that may be more common than direct antifungal drug resistance. Fifth, an uninterrupted initial induction phase of combination antifungal therapy is important, and in fragile patients, lipid products of amphotericin B may be more effective at keeping therapy on schedule than the conventional amphotericin B preparation. Sixth, because of differences in resources, there are health care systems without access to certain polyenes and flucytosine; therefore, in these situations, it is important to apply the best pharmacodynamic strategies with the available resources. Finally, despite many immunological studies and the use of recombinant cytokine proteins in humans (20), immunotherapy such as IFN- $\gamma$ is still recommended as an alternative rather than a primary therapy.

\section{Future directions}

In cryptococcosis, our goal is to prevent or effectively treat the fungal disease so that a patient's outcome is determined by the underlying disease, not the secondary infection. In some respects, we have made progress. At our own institution, survival rates of cryptococcal meningitis are better for HIV-infected and transplant recipient patients compared with those of non-HIVinfected, non-transplant patients. This observation likely reflects our ability to effectively treat the fungal disease and to better control the underlying diseases or conditions; however, there is still much work to do. The mortality rate for crypto- coccal meningitis among our transplant recipients and HIV-infected patients is still approximately $16 \%$ and is $31 \%$ among non-HIV-infected, non-transplant patients (21). We must do better. Despite the wonderful progress in clinical management over the past 20 years, improved immunological understanding, and molecular biology investigations, the sugar-coated yeast whose sweetness sickens continues to represent the perfect model system to fundamentally understand infectious diseases through both the host and the microbe. It takes "two to tango," and while the hosts bring many important factors that determine disease outcome, so do the cryptococcal yeasts. The work of Sabiiti et al. (8) clearly demonstrates that not all cryptococcal strains are created equally, and like many infectious diseases, we need to understand the interplay between hosts and pathogens.

\section{Acknowledgments}

This work is supported by Public Health Service Grants AI73896, HL30923, and AI93257.

Address correspondence to: John R. Perfect, Hanes House, Trent Drive, Duke University Medical Center, Durham, North Carolina 27710, USA. Phone: 919.684.6854; Fax: 919.684.8902; E-mail: Perfe001@mc.duke.edu.

1. Kaufman L, Blumer S. Cryptococcosis: The awakening giant. Presented at: Proceedings of the Fourth International Conference on Mycoses; June 1977; Brasilia, Brazil. PAHO Scientific Publication no. 356

2. Park BJ, Wannemuehler KA, Marston BJ, Govender N, Pappas PG, Chiller TM. Estimation of the current global burden of cryptococcal meningitis among persons living with HIV. AIDS. 2009;23(4):525-530.

3. Byrnes EJ 3rd, et al. Emergence and pathogenicity of highly virulent Cryptococcus gattii genotypes in the northwest United States. PLoS Pathog. 2010;6(4):e1000850.

4. Singh N, Perfect JR. Immune reconstitution syndrome associated with opportunistic mycoses. Lancet Infect Dis. 2007;7(6):395-401.

5. Rohatgi $\mathrm{S}$, et al. $\mathrm{Fc} \gamma$ receptor $3 \mathrm{~A}$ polymorphism and risk for HIV-associated cryptococcal disease. MBio. 2013;4(5):e00573-13.

6. Rosen LB, et al. Anti-GM-CSF autoantibodies in patients with cryptococcal meningitis. J Immunol. 2013;190(8):3959-3966.

7. Boulware D, et al. Clinical features and serum biomakers in HIV immune reconstitution inflammatory syndrome after cryptococcal meningitis: aprespective cohort study. PLoS Med. 2010;7(12):e1000384.

8. Sabiiti W, et al. Efficient phagocytosis and laccase activity affect the outcome of HIV-associated cryptococcosis. J Clin Invest. 2014;124(5):2000-2008.

9. Robertson EJ, et al. Cryptococcus neoformans ex vivo capsule size is associated with intracranial pressure and host immune response in HIVassociated cryptococcal meningitis. J Infect Dis. 2014;209(1):74-82.

10. Ormerod KL, et al. Comparative genomics of serial isolates of Cryptococcus neoformans reveals gene associated with carbon utilization and virulence. G3 (Bethesda). 2013;3(4):675-686.

11. Janbo G, et al. The genome sequence of Cryptococcus neoformans var. grubii reveals complex mechanism of RNA expression and virulene plasticity. PLoS Genet. In press.

12. Hu G, et al. Variation in chromosome copy number influences the virulence of Cryptococcus neoformans and occurs in isolates from AIDS patients. BMC Genomics. 2011;12:256.

13. Chen Y, et al. The Cryptococcus neoformans transcriptome at the site of human meningitis. MBio. 2014;5(1):e01087-13.

14. Hansen J, et al. Large-scale evaluation of the immuno-mycologics lateral flow and enzyme-linked immunoassays for detection of cryptococcal antigen in serum and cerebrospinal fluid. Clin Vaccine Immunol. 2013;20(1):52-55.

15. Bicanic $\mathrm{T}$, et al. Independent association between rate of clearance of infection and clinical outcome of HIV-associated cryptococcal meningitis: analysis of a combined cohort of 262 patients. Clin Infect Dis. 2009;49(5):702-709.

16. Jarvis JN, et al. Determinants of mortality in a combined cohort of 501 patients with hiv-associated cryptococcal meningitis: implications for improving outcomes. Clin Infect. 2014;58(5):736-745.

17. Day JN, et al. Combination antifungal therapy for cryptococcal meningitis. $N$ Engl J Med. 2013; 368(14):1291-1302.

18. Perfect JR, et al. Clinical practice guidelines for the management of cryptococcal disease: 2010 update by the infectious diseases society of America. Clin Infect Dis. 2010;50(3):291-322.

19. Bratton EW, et al. Approaches to antifungal therapies and their effectiveness among patients with cryptococcosis. Antimicrob Agents Chemother. 2013;57(6):2485-2495.

20. Jarvis JN, et al. Adjunctive interferon-y immnotherapy for the treatment of HIV-associated cryptococcal meningitis: a randomized controlled trial. AIDS. 2012;26(9):1105-1113.

21. Bratton E, et al. Comparison and temporal trends of three groups with cryptococcosis: HIV-infected, solid organ transplant, and HIV-negative/nontransplant. PLoS One. 2012;7(8):e43582. 